\title{
Diurnal Profile of Gallbladder Size in Diabetic Patients: Ultrasonographic Evaluation of Diabetic Neurogenic Gallbladder
}

\author{
Hiroyoshi Onodera, Hiraku Sugawara, Toru Hirata, \\ Nobuyuki Imai, Akio Nagasaki, Binkoh Yoda, Takayoshi \\ Tоуота and Yoshio Goto \\ Third Department of Internal Medicine, Tohoku University \\ School of Medicine, Sendai 980
}

\begin{abstract}
Onodera, H., Sugawara, H., Hirata, T., Imai, N., Nagasaki, A., Yoda, B., Точота, T. and Goto, Y. Diurnal Profile of Gallbladder Size in Diabetic Patients: Ultrasonographic Evaluation of Diabetic Neurogenic Gallbladder. Tohoku J. exp. Med., 1983, 139 (2), 179-186 — The diurnal change of gallbladder size was clearly measured with a real time ultrasonography. To visualize the gallbladder, 21 diabetic patients and 11 healthy subjects were scanned right subcostally in the supine position nine times between 8:00 a.m. and 8:00 p.m. Photographs of the images were produced on Polaroid film, and the area of gallbladder was calculated. The diurnal changes of the gallbladder size were expressed as the percentage of the size measured at 8:00 a.m. after an overnight fast. The gallbladder in normal control started contracting immediately after breakfast and reached $57.2 \pm 7.6 \%$ at 9:00 a.m. In the diabetic patients with and without autonomic neuropathy the gallbladder contracted to $80.9 \pm 14.5 \%$ and $72.4 \pm$ $14.8 \%$, respectively, at 9:00 a.m. while the nadir of the diurnal profile curve in healthy subjects was $24.5 \pm 3.6 \%$ at $3: 00$ p.m., in the diabetic patients with and without autonomic neuropathy those were $57.7 \pm 6.1 \%$ at $6: 00$ p.m. and $41.3 \pm 8.3 \%$ at 8:00 p.m., respectively. This implies that the gallbladder contraction was delayed and poor in diabetic patients. In this paper, the gallbladder tonicity index is proposed, which is useful for the easy evaluation of autonomic state of gallbladder in diabetes mellitus. diabetes mellitus; diabetic autonomic neuropathy; gallbladder; ultrasonography; gallbladder tonicity index
\end{abstract}

The occurrence of "diabetic neurogenic gallbladder" syndrome in diabetic patients is characterized by an enlarged gallbladder, poor contraction and/or poor visualization of the gallbladder and the absence of symptoms suggesting gallbladder disease (Gitelson et al. 1963). The size of the diabetic neurogenic gallbladder is reported to be more than three to nine times greater than that of the normal condition (Gitelson et al. 1969; Bloom and Stachenfeld 1969; Clarke et al. 1979) Gallbladder hypotony is more frequently found in diabetic patients (Grodzki et al. 1968; Rigberg and Glouberman 1977).

In recent years, gallbladder function tests have been performed by employing real time ultrasonography (Palframan and Meire 1979; Suzuki 1980; Kinugasa

Received for publication, May 7, 1982. 
et al. 1981; Ochiai et al. 1981). Marumo et al. (1981) reported poor gallbladder contraction in diabetic patients when real time ultrasonography was performed within $60 \mathrm{~min}$ after the intake of yolks.

We investigated the diurnal changes of gallbladder size in diabetic patients with and without autonomic neuropathy with real time ultrasonography to clarify the autonomic state of diabetic gallbladder.

\section{Materials and Methods}

Nine diabetic patients ranging from 15 to 62 years in age (40.4 \pm 14.5 , mean \pm s.D.) with autonomic neuropathy and 12 diabetic patients between the ages of 15 and 71 years $(46.3 \pm 17.3)$ without autonomic neuropathy were examined. The patients in the former group had at least one symptom and/or sign of autonomic neuropathy, i.e., nocturnal diarrhea, constipation, sphincter disturbance, delayed or absence of pupil light reflex, orthostatic hypotention, sudomotor disturbance and impotence. Eleven nondiabetic volunteers, ranging from 26 to 59 years in age $(44.0 \pm 15.1)$, without history nor symptoms of gallbladder diseases served as the control.

To visualize the gallbladder, real time ultrasonography with a $3.5 \mathrm{MHz}$ probe (Model SAL-20A, Toshiba) was used. The subjects were scanned right subcostally in the supine position nine times between 8:00 a.m. and 8:00 p.m. (at 8, 9, 10:30, 12, 13, 15, 17, 18 and 20 o'clock). Meals were served immediately after the 8, 12 and 17 o'clock examinations, respectively. No cholecystokinetic drug was used. Photographs of the images were produced on Polaroid film (No. 611), and the area of gallbladder was calculated with ID Data Tablet/Digitizer (Summergraphics Corp. USA). The diurnal change of gallbladder size was expressed as the percentage of the size measured at 8:00 a.m. after overnight fasting.

In six patients gastric emptying was measured using the dye method described by Routh et al. (1967). The correlation between gallbladder contraction and the rate of gastric emptying was studied.

Means \pm standard deviations (s.D.) of the gallbladder size were calculated, and statistical analyses were carried out according to the Student's $t$-test.

\section{RESULTS}

The gallbladder in the control started contracting immediately after breakfast and reached $57.2 \pm 7.6 \%$ at 9 o'clock. In diabetic patients the contraction was $76.3 \pm 14.9 \%$ at 9 o'clock. The nadir of the diurnal profile curve in the control was $24.5 \pm 3.6 \%$ at 15 o'clock, while that of the diabetic patients was $49.1 \pm 16.6 \%$ at 18 o'clock (Fig. 1).

Gallbladder contraction was poorer in diabetic patients with autonomic neuropathy than in those without (Fig. 2). In diabetic patients with and without autonomic neuropathy, the gallbladder contracted to $80.9 \pm 14.5 \%$ and $72.4 \pm 14.8$ $\%$ at 9 o'clock, respectively. The nadir of the diurnal profile curve in diabetic patients without autonomic neuropathy was $41.3 \pm 8.3 \%$ at 20 o'clock. In diabetic autonomic neuropathy it was $57.7 \pm 6.1 \%$ at 18 o'clock.

The mean minimal size at the most contracted state in diabetic patiens with and without autonomic neuropathy were $51.0 \pm 5.6 \%$ and $34.0 \pm 8.3 \%$, respectively, and that of the control was $22.2 \pm 3.5 \%$ (Fig. 3).

The correlation between the gastric emptying and the minimal gallbladder size 


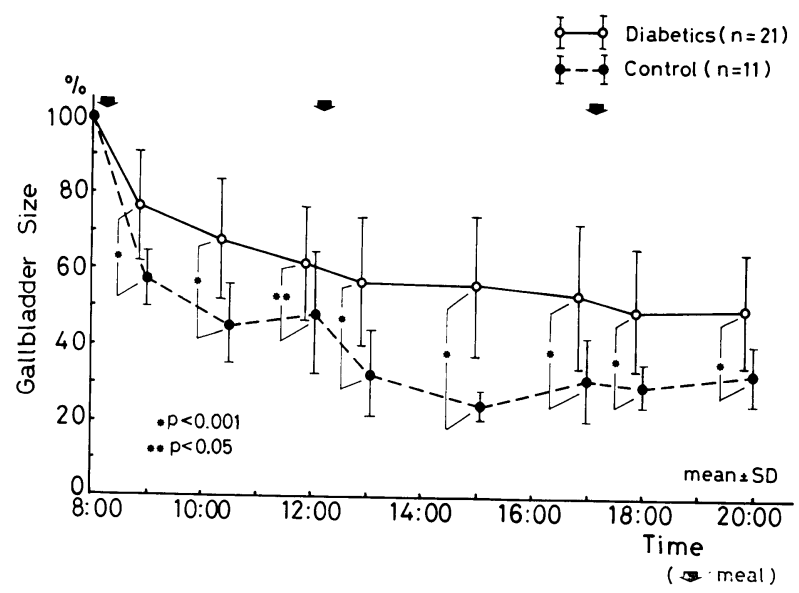

Fig. 1. Diurnal profile of the gallbladder size. The gallbladder contraction of diabetic patients is delayed and poor in comparison with the control.

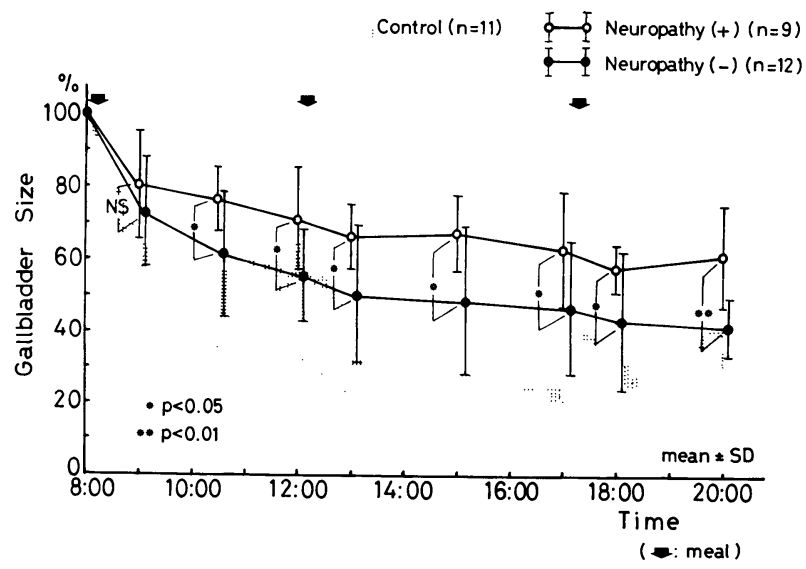

Fig. 2. Diurnal profile of the gallbladder size in diabetic patients. The gallbladder contraction of diabetic patients with autonomic neuropathy is poor in comparison with that of diabetic patients without autonomic neuropathy.

was significant $(r=0.92, p<0.02)$ (Fig. 4). The more gastric emptying was delayed, the poorer the gallbladder contraction was.

\section{Case Report}

Case 1. This 40 years-old male has been suffering from diabetes mellitus for the past 15 years, and had had complications including diabetic retinopathy for 10 years, diabetic cataract for 3 years and incontinence of both stool and urine for 2 years. He had diarrhea several times a day. Motor nerve conduction velocity was delayed. The rate of gastric emptying was impaired. Roentgen examination of the stomach after overnight fasting revealed the dilated stomach 


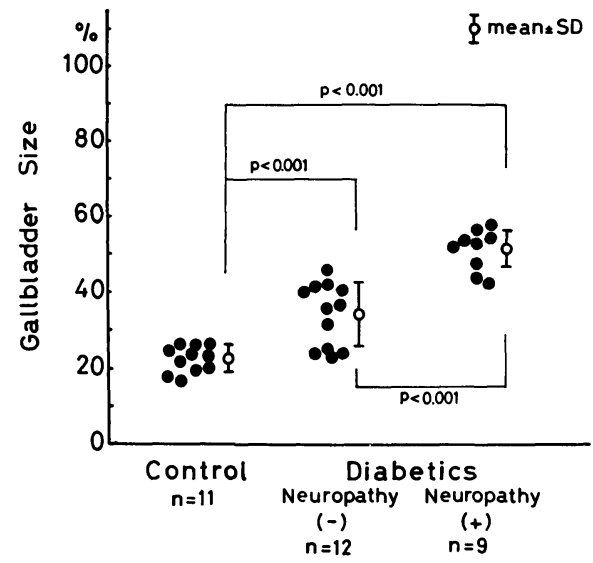

Fig. 3. Minimal gallbladder size. The gallbladder sizes of the most contracted state in controls and diabetic patients with and without autonomic neuropathy. Significant statistical differences were shown among the three groups.

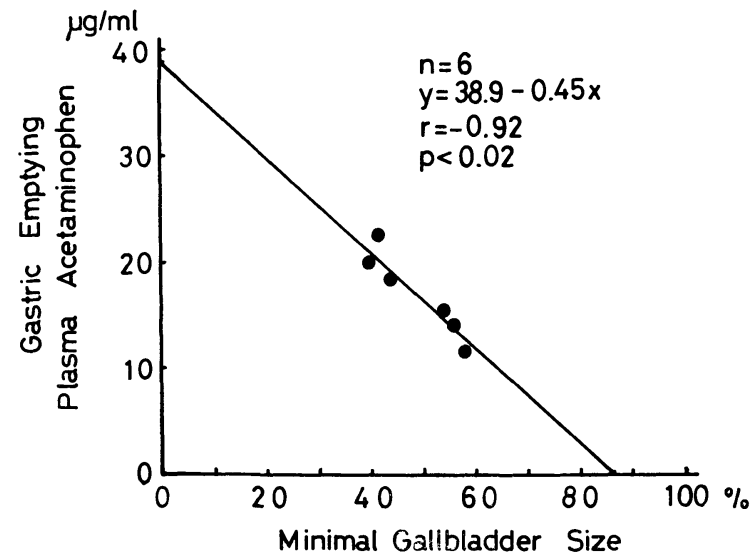

Fig. 4. Correlation between gastric emptying and the minimal gallbladder size in six diabetic patients. The more gastric emptying delay, the poorer the gallbladder contraction is.

and a large amount of food residue (Fig. 5). The diurnal profile of the gallbladder size showed poor contraction (Fig. 6).

Case 2. This 38 years-old female has been suffering from diabetes mellitus for the past 17 years. Twelve years ago, after the second delivery, axillary and public hair became scanty, her skin became thin, dry and pale and amenorrhea appeared. She was referred to our clinic for further examinations. Endocrine function tests revealed Sheehan syndrome. Roentgen examination of the stomach after overnight fasting showed the food residue (Fig. 7). The diurnal profile showed poor contraction of the gallbladder (Fig. 8). 


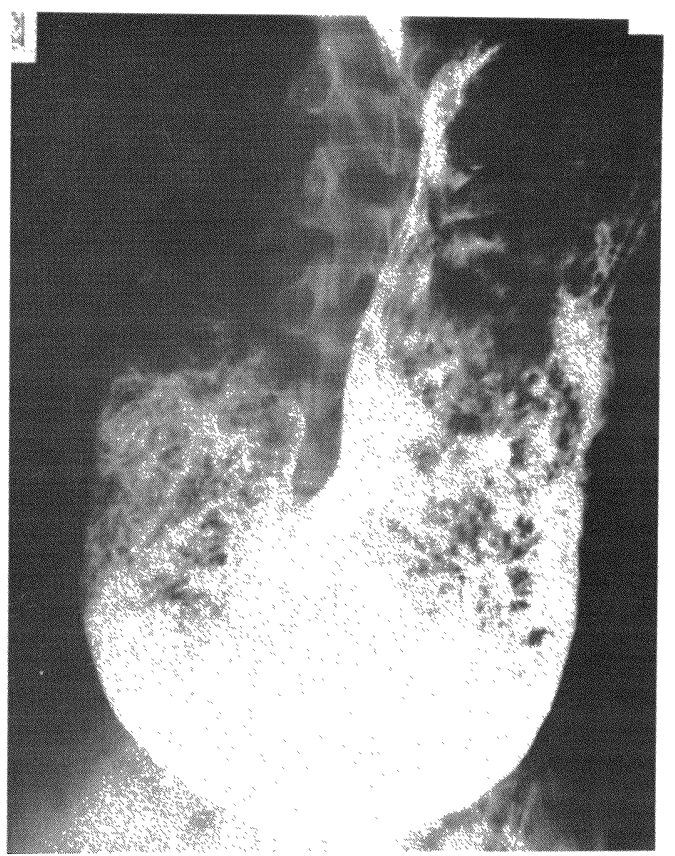

Fig. 5. Roentgenogram of the stomach in Case 1 showing the dilated stomach and a large amount of food residue as a manifestation of diabetic gastroenteropathy.

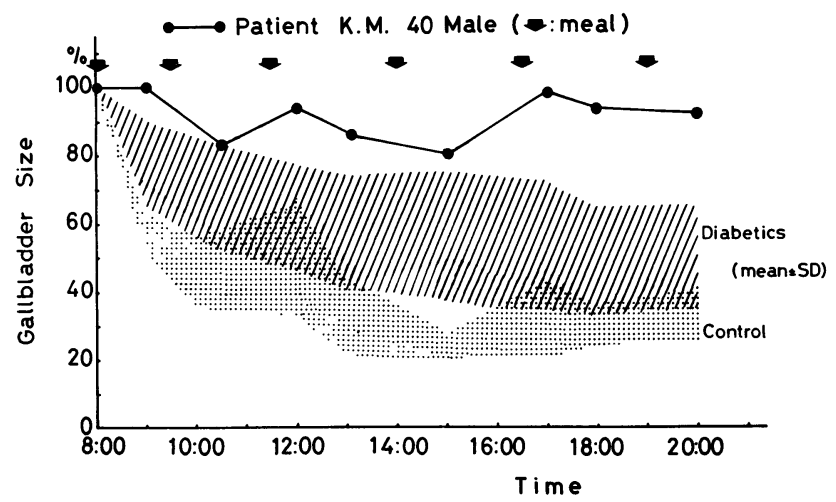

Fig. 6. Diurnal profile of the gallbladder size in Case 1. Poor gallbladder contraction is shown.

\section{Discussion}

The mean surface area of the gallbladder, measured from $\mathrm{X}$-ray shadows, in diabetic patients is significantly larger than that in nondiabetic individuals (Gitelson et al. 1969). In some diabetic patients the large gallbladder may be a manifestation of biliary dyskinesia due to autonomic neuropathy and is sometimes called "diabetic neurogenic gallbladder" (Gitelson et al. 1963). Bloom and Stachenfeld (1969) reported that four $(16 \%)$ out of 25 diabetic patients had 


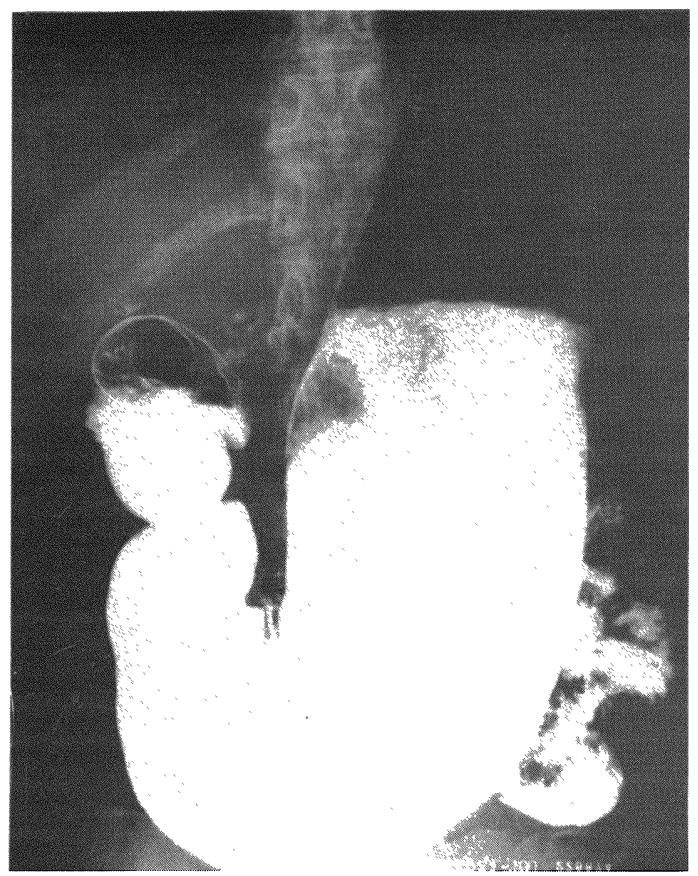

Fig. 7. Roentgenogram of the stomach in Case 2 showing the food residue.

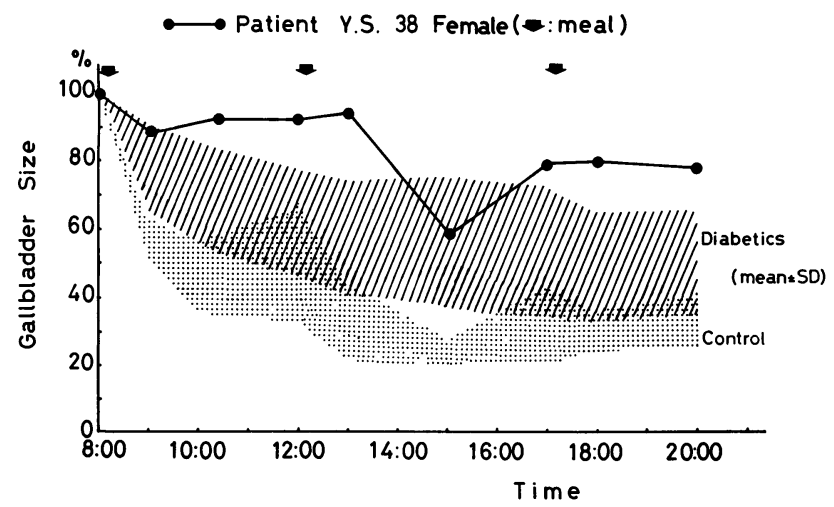

Fig. 8. Diurnal profile of the gallbladder size in Case 2. Poor gallbladder contraction is shown.

enlarged gallbladder and the condition resembled cholecystomegaly induced by vagotomy. On the contrary, Grodzki et al. (1968) reported that the mean surface of the gallbladder in diabetic patients was slightly smaller, although not significantly, than in control. He also reported that the average contractility of the gallbladder was definitely lower in diabetic patients.

Ultrasonographical examination of the kinetics of the gallbladder contraction has become satisfactory method (Palframan and Meine 1979; Suzuki 1980; 
Kinugasa et al. 1981; Ochiai et al. 1981). Marumo et al. (1981) in their study using real time ultrasonography reported poor contraction of the gallbladder in diabetic patients within $60 \mathrm{~min}$ after intake of yolks.

Ultrasonography is superior to X-ray cholecystography and other methods since ultrasonography is non-invasive, harmless and does not rely on the contrast media metabolism. Accordingly it is easy to obtain the serial change of the gallbladder size with real time ultrasonography.

Our study on the serial change of gallbladder size (diurnal profile of gallbladder size) demonstrated that the contraction of the gallbladder was delayed and poor in diabetic patients.

One important observation on the diurnal profile curve was the gallbladder size at 9 o'clock, about $1 \mathrm{hr}$ after breakfast. In the control the gallbladder immediately contracted to $57.2 \pm 7.6 \%$ at 9 o'clock, while in diabetic patients the gallbladder poorly contracted to $76.3 \pm 14.9 \%$.

Another important observation is the gallbladder size at 15 o'clock. In the control the nadir of the diurnal profile curve was $24.5 \pm 3.6 \%$ (ranging from 19.2 to $30.7 \%$ ) at 15 o'clock, but in diabetic patients the gallbladder size at 15 o'clock was not the nadir and was significantly larger than that of the control.

For a simple evaluation of the autonomic gallbladder state in diabetes mellitus, the sum of the gallbladder sizes at 9 and 15 o'clock is proposed as a gallbladder tonicity index. The correlation between the minimal gallbladder size in diurnal profile and the index was significant $(r=0.83, p<0.001)$ (Fig. 9). The index in the control was $81.6 \pm 8.4$ and those in diabetic patients with and without autonomic neuropathy were $147.9 \pm 21.1$ and $120.2 \pm 20.8$, respectively

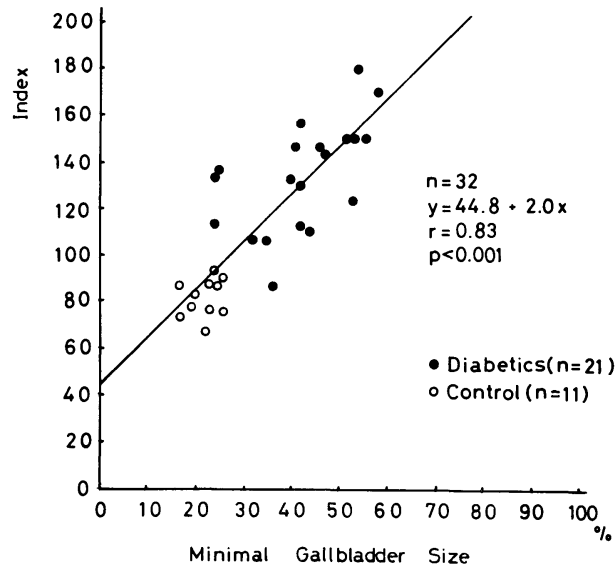

Fig. 9

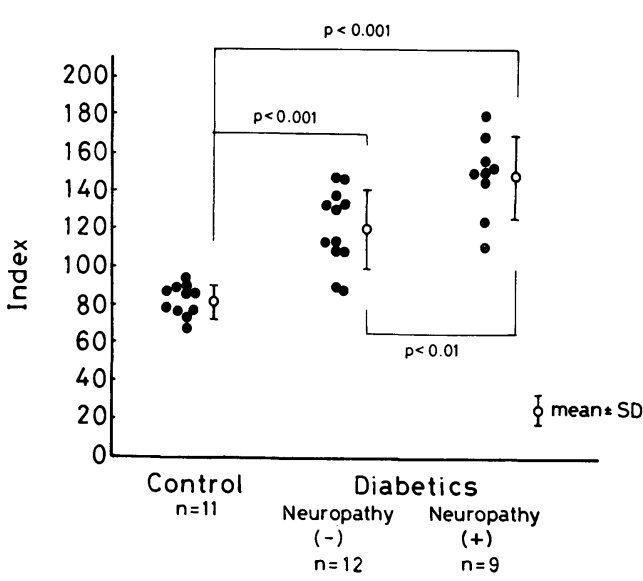

Fig. 10

Fig. 9. Correlation between gallbladder tonicity index and minimal gallbladder size. The significant correlation is shown.

Fig. 10. The gallbladder tonicity index, the sum of the gallbladder sizes at 9 and 15 o'clock. Significant statistical differences are shown among the three groups. 
(Fig. 10). The differences among the indeces of three groups were statistically significant.

The diurnal profile of gallbladder size is valuable for the diagnosis of the diabetic neurogenic gallbladder. And the gallbladder tonicity index is useful as a simple evaluation method for autonomic neuropathy.

\section{References}

1) Bloom, A.A. \& Stachenfeld, R. (1969) Diabetic cholecystomegaly. J. Amer. med. Ass. 208, 357-359.

2) Clarke, B.F., Ewing, D.J. \& Campbell, I.W. (1979) Diabetic autonomic neuropathy. Diabetologia, 17, 195-212.

3) Gitelson, S., Schwartz, A., Frankel, M. \& Chowers, I. (1963) Gallbladder dysfunction in diabetes mellitus. Diabetes, 12, 308-312.

4) Gitelson, S., Oppenheim, D. \& Schwartz, A. (1969) Size of the gallbladder in patients with diabetes mellitus. Diabetes, 18, 493-498.

5) Grodzki, M., Mazurkiewicz-Rozynska, E. \& Czyzyk, A. (1968) Diabetic cholecystopathy. Diabetologia, 4, 345-348.

6) Kinugasa, T., Sekimoto, K., Fukano, S., Iwagaki, S., Masuda, K. \& Saito, Y. (198I) Measurement of gallbladder size in contraction by real time ultrasonography. JSUM Proceedings, 38, 381-382. (in Japanese with English abstract)

7) Marumo, K., Hayashi, M., Fujii, S., Seki, J., Wada, M. \& Asai, H. (1981) Gallbladder function in diabetics with ultrasonography. JSUM Proceedings, 38, 383-384. (in Japanese with English abstract)

8) Ochiai, Y., Tomiyama, F., Itoh, T., Shinagawa, A., Ono, Y., Kawashima, Y. \& Honda, T. (1981) Studies of the gallbladder contraction motion by electric linear scanning. JSUM Proceedings, 38, 385-386. (in Japanese with English abstract)

9) Palframan, A. \& Meire, H.B. (1979) Real-time ultrasound. A new method for studying gall-bladder kinetics. Brit. J. Radiol., 52, 801-803.

10) Rigberg, L. \& Glouberman, S. (1977) Diabetes mellitus and the gastrointestinal tract. Part II. Small and large intestine and gallbladder. Arizona Med., 34, 174-175.

11) Routh, J.I., Shane, N.A., Arredondo, E.G. \& Paul, W.D. (1967) Method for the determination of acetylsalicylic acid in the blood. Clin. Chem., 13, 734-739.

12) Suzuki, T. (1980) Clinical significance of the gallbladder enlargement and contraction studied by ultrasonography. Jap. J. Gastroent., 77, 415-422. (in Japanese with English abstract) 